\title{
Features of discrete VLF emissions observed at Gulmarg, India during the magnetic storm of 6-7 March, 1986
}

\author{
R Singh ${ }^{1}$, A K Singhi,*, D SiIngh ${ }^{3,4}$ and R P Singh ${ }^{2}$ \\ ${ }^{1}$ Indian Institute of Geomagnetism, New Panwel, Navi Mumbai 410 218, India. \\ ${ }^{2}$ Department of Physics, Banaras Hindu University, Varanasi 221 005, India. \\ ${ }^{3}$ Indian Institute of Tropical Meteorology, Pune 411 008, India. \\ ${ }^{4}$ Institute of Environmental Physics, University of Tartu, 18, Ulikooli Street, Tartu 50090, Estonia. \\ *e-mail: abhay_s@rediffmail.com
}

During the analysis of archived VLF data from Indian low latitude ground stations, some discrete VLF emissions recorded at the low latitude ground station Gulmarg (geomagnetic latitude $24^{\circ} 26^{\prime} \mathrm{N}$; geomagnetic longitude $\left.147^{\circ} 09^{\prime} \mathrm{E}, \mathrm{L}=1.28\right)$ during moderate magnetic storm activity $\left(\sum K_{P}^{-}=32\right.$, $K_{P}$ index varies from 4 to 6 during the observation period) on 6/7 March, 1986 are presented in this paper. The dynamic spectra of these discrete VLF emissions were observed along with tweeks and its harmonics, which is interesting and complex to explain. In most of the events the harmonic frequency of tweeks correlates with the starting frequency of harmonics of discrete emissions. In order to explain the observed features of discrete VLF emissions, we propose cyclotron resonance interaction between whistler mode wave and energetic electrons of inner radiation belt as possible generation mechanism. An attempt is also made to determine parallel energy, anisotropy and wave growth relevant to the generation process of VLF emissions.

\section{Introduction}

Magnetospheric ELF/VLF emissions is a class of natural radio phenomena, which is often observed in close association with whistlers both at the Earth's surface and onboard satellites. They are grouped as: (a) unstructured continuous emissions in both time and frequency which tend to maintain a steady state like hiss, resonance band and noise bands near the ion gyrofrequencies, and (b) structured discrete emissions with a repetitive and even periodic character which tend to be transient like chorus, periodic emissions and other transient discrete emissions such as hooks, risers, fallers, pseudo whistlers (Helliwell 1967; Hattori et al 1991; Sazhin and Hayakawa 1992; Bell et al 2000). The generation of discrete VLF emissions is one of the most puzzling problems of VLF waves in the Earth's magnetosphere. Although it is generally believed that their generation is connected with the cyclotron resonance of whistlermode waves and radiation belt electrons (Helliwell 1967), the mechanism responsible for the origin of discrete VLF emissions and the formation of spectrum of separate elements are still a subject of active experimental and theoretical research (Trakhtengerts 1999; Santolik and Gurnett 2003; Santolik et al 2004; Singh and Singh 2004 with references therein). At mid- and high-latitudes the observed correlation between discrete VLF emissions and energetic electrons suggests the key role of the latter in the generation of the former. Nunn and Sazhin (1991) considered interaction between VLF hiss and energetic electrons and explained the similarities between fine structure of chorus and hiss emissions. To explain the spectrogram of hiss and discrete VLF emissions, Dowden (1971) suggested that whistlers echoing in the discrete emission path are initiated by sferics and appear to be the source of discrete VLF emissions and

Keywords. Magnetospheric physics; space plasma physics; wave particle interactions; waves and instabilities; VLF emissions; upper atmosphere. 


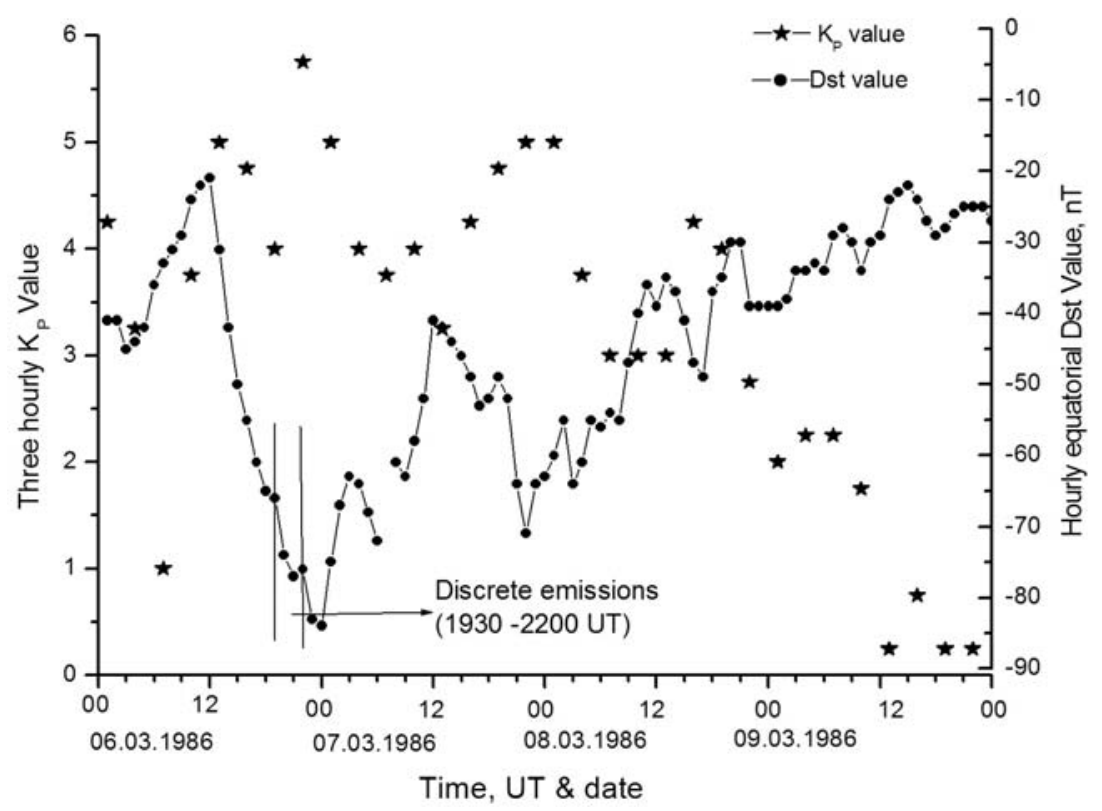

Figure 1. Equatorial hourly $D$ st variations and three hourly $K_{P}$ index during the main phase magnetic storm 6-9 March, 1986. The emission period of the event is also marked.

hiss bursts involving lightning triggered from the Earth's magnetosphere.

Since the propagation characteristics of ELF/ VLF emissions in the low latitude ionosphere are not properly known, the mechanism of their generation source and propagation are far from being well understood (Singh et al 2000; Singh and Singh 2004). Therefore, a better understanding of the generation mechanism for ELF/VLF emissions observed at low latitudes would be useful for analyzing the properties of high energy trapped electrons. During the course of our analysis of old ELF/VLF data recorded during January 1986 to July 1986 at low latitude ground station Gulmarg (geomag. lat. $24^{\circ} 26^{\prime} \mathrm{N}$; geomag. long. $147^{\circ} 09^{\prime} \mathrm{E}, \mathrm{L}=1.28$ ), we found a new type of discrete VLF emission which we report here with a discussion of their probable generation mechanism.

In the present paper, first we present spectral analysis of the ELF/VLF discrete emission events observed at Gulmarg during the routine recording of whistlers. The dynamic spectra of the discrete VLF emissions were observed along with tweeks and its harmonics. In most of the events, the lowest frequency of harmonics of discrete emissions correlates with the second and third harmonic frequency of tweeks. Further, generation mechanism of these discrete VLF emissions has been proposed and an attempt is made to determine parallel energy, anisotropy and wave growth relevant to the generation process of discrete emissions. Finally results are discussed with other published results.

\section{Experimental observations and analysis}

At the low-latitude ground station Gulmarg, the wideband ELF/VLF waves were received by a T-type antenna, suitably amplified by pre- and main-amplifiers and recorded using a tape recorder. The recorded data were analysed by digital Sonograph machine and Advance VLF Data Analysis System (AVDAS). At low latitudes, nights with observable ELF/VLF emissions are rather rare, and the activity is closely related to strong magnetic activity (Siingh et al 2005). Several interesting ELF/VLF events were recorded during a magnetic storm often large enough to allow for a statistical analysis. The observations of discrete VLF emissions at low-latitude ground station Gulmarg are unusual in the sense that most of the reported discrete emissions are from satellites (Cornilleau-Wehrlin et al 1978; Hattori et al 1991; Santolik and Gurnett 2003; Santolik et al 2004). In this paper, the discrete VLF emissions recorded during the night of 6-8 March, 1986 are analysed. We have recorded a new type of discrete VLF emission, which is associated with the tweaks and its harmonics. These discrete VLF emissions were recorded during the magnetic storm period of 6-9 March, 1986, with minimum Dst index of $-84 \mathrm{nT}$ on 6 March and maximum $\sum K_{p}=34$ on 7 March. The Dst-index variations and $K_{p}$-index variations are shown in figure 1 , in which the observation period of the event is also marked. It is to be noted that these discrete events were observed during the main phase of the magnetic storm. 
07 March, 1986 GULMARG

Geomag. Lat. $24^{\circ} 26^{\prime} \mathrm{N}$, Long. $147^{\circ} 09^{\prime} \mathrm{E}$

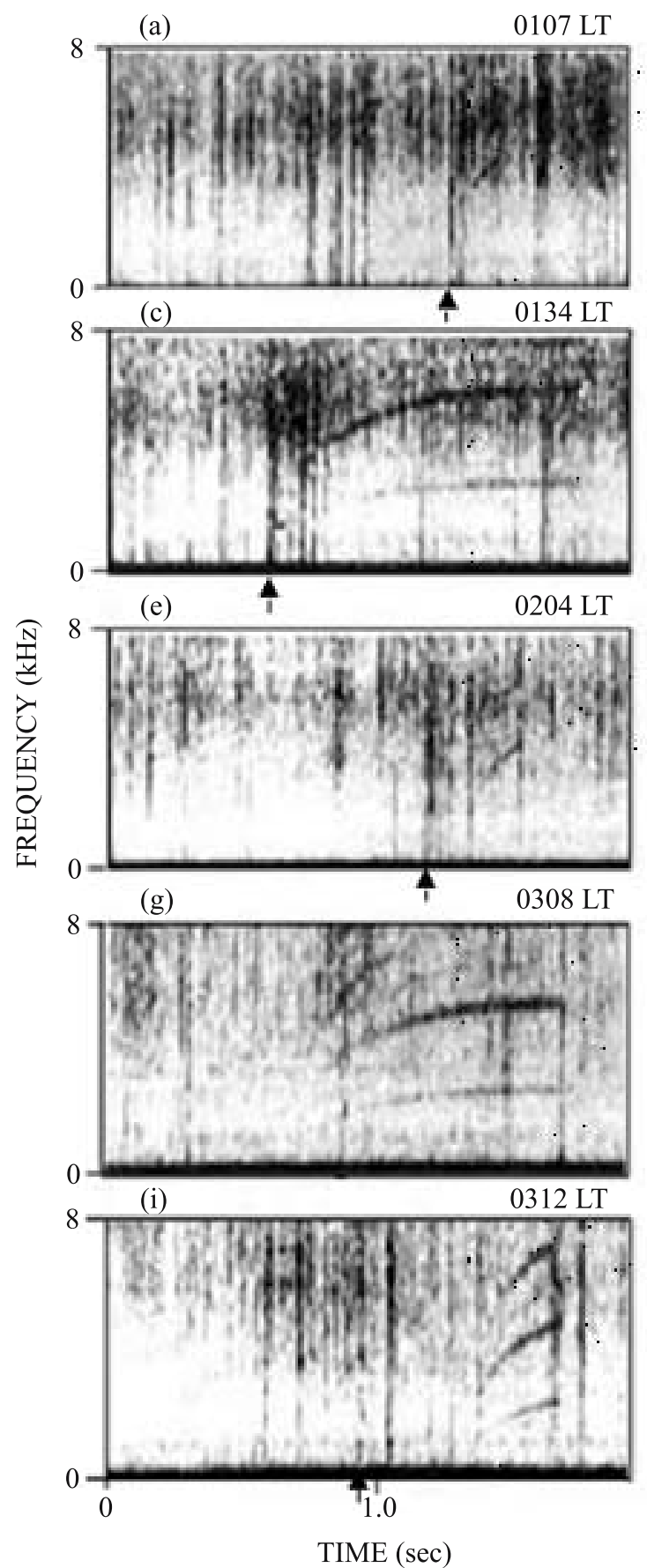

(b)
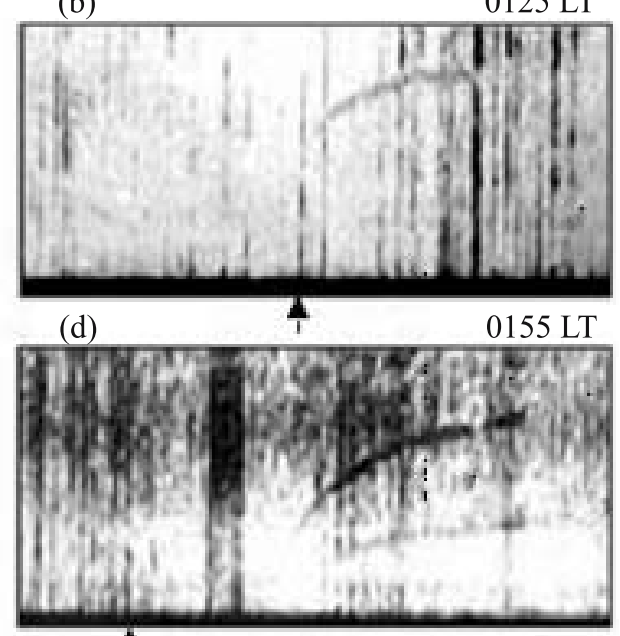

(f) 4

0205 LT

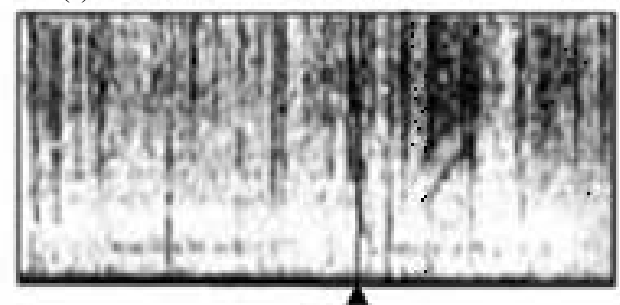

(h)
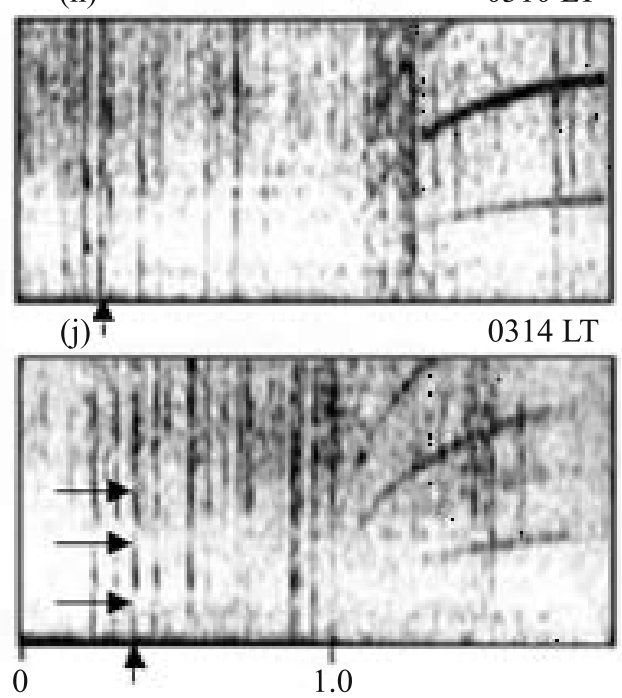

TIME (sec)

Figure 2. Typical examples of lightning generated sferices alongwith discrete VLF emissions observed at Gulmarg on 7 March, 1986 (local time). Arrows mark the tweeks. The first, second and third harmonics of the tweeks are also marked in (j) by arrows on frequency scale.

\subsection{Spectral analysis of discrete $V L F$ emissions}

Typical frequency-time spectrograms of sferics and discrete VLF emissions recorded at Gulmarg during the night hours of 6-7 March, 1986 are shown in figure 2. Discrete emission events started at
$1930 \mathrm{UT}$ (0100 LT) and continued up to $2200 \mathrm{UT}$ (0330 LT). Figure 2 shows an association between VLF spherics (tweeks) and discrete VLF emissions. Here we note that for most of the events the fundamental frequency of tweeks corresponds to the initiating frequency of fundamental discrete emissions. The second harmonic frequency of tweeks 
corresponds to the starting frequency of the second harmonics of discrete emissions and so on. Tweeks are ELF/VLF electromagnetic waves which originated in the distant lightning and propagated in the Earth-ionosphere waveguide. They are known to exhibit remarkable dispersion near the cut-off frequencies of the first-, second-, third-order mode waves, and these dispersion effects are found to fit well with the Earth-ionosphere waveguide propagation theory (Singh and Singh 1996). In the course of time the upper boundary frequency of discrete VLF emissions was fluctuating and the number of events increased with the passage of time. First harmonic tweeks (sferics) accompanied with the single event of discrete VLF emissions observed in different frequency ranges between 2.6 and $6.5 \mathrm{kHz}$ is shown in figure $2(\mathrm{a}, \mathrm{b}$ and $\mathrm{e})$, whereas figure $2(\mathrm{c}$, $\mathrm{d}$ and f) contain second harmonic tweeks and correspondingly two discrete VLF emission events lying in different frequency ranges between 2 and $6.5 \mathrm{kHz}$. Figure $2(\mathrm{~g}, \mathrm{~h}, \mathrm{i}$ and $\mathrm{j})$ clearly show third harmonic tweeks accompanied by three events of discrete VLF emissions of short and long duration in different frequency ranges between 1 and $8 \mathrm{kHz}$. Hence, a fairly consistent pattern emerges from the observed features of the tweeks and discrete VLF emissions recorded at Gulmarg and a correlation is found between them.

From figure 2, an interesting point emerges concerning the correspondence between the cut-off frequencies of harmonics of tweeks and starting frequency of corresponding discrete emissions. For example, figure $2(\mathrm{j})$ shows that the first harmonic frequency is $0.7 \mathrm{kHz}$ and the initiating of discrete emission is $0.7 \mathrm{kHz}$. Similarly the cut-off frequency of the second harmonic is $1.9 \mathrm{kHz}$ and corresponding starting frequency of the second discrete VLF emission is $1.9 \mathrm{kHz}$. Corresponding frequency for the third harmonic and the third discrete emission are $3.4 \mathrm{kHz}$ and $3.4 \mathrm{kHz}$ respectively. The observed discrete VLF emission elements have the following mean parameters: $f_{\min }=1 \mathrm{kHz}, f_{U B}=f_{\max }=$ $5.04 \mathrm{kHz}$ and $d f / d t=6.622 \mathrm{~Hz} \mathrm{sec}{ }^{-1}$. The standard deviation of $f_{U B}$ was found to be about 0.65 . The discrete VLF emissions shown in figure 2 occurred in the wide frequency range between 1 and $8 \mathrm{kHz}$ with the rate of change of frequency with time $(d f / d t)$ between 0.9 and $10.5 \mathrm{kHz} \mathrm{sec}{ }^{-1}$. The relationship between the cut-off of the tweak and the cut-off of the VLF emissions indicates that the emissions may have actually propagated in the Earth-ionosphere wave-guide and this correlation in frequency could be due to wave-guide cut-off.

\section{Generation mechanism}

The dynamic spectrum of tweeks (sferics) and its higher harmonics can be understood by considering part of VLF energy after lightning discharge to propagate through the Earthionosphere waveguide (Singh and Singh 1996; Shvets and Hayakawa 1998). Discrete VLF emissions can be explained by transverse resonance interaction between whistler mode waves and counter streaming energetic electrons (Helliwell 1967; Nunn and Sazhin 1991). Analyzing Ariel 3 and 4 satellite data Hayakawa (1989) has also suggested that low latitude VLF emissions may have originated during lightning discharges.

The observed features of tweeks shown in figure 2 and cut off frequency of tweeks confirm that the scenario in which lightning source is closer to the observation point in the northern hemisphere may give rise to higher harmonics. Usually lightning energy is coupled to the Earth-ionosphere wave-guide and tweeks along with the higher harmonics are generated. Only in rare cases, lightning generated electromagnetic energy propagates both along the geomagnetic field lines and in the Earthionosphere waveguide, for which tweeks are found to be correlated with VLF whistler-mode waves. The second and the third harmonic of discrete VLF emissions may have been generated through the wave-particle interactions in the magnetosphere. The two events having their origin in the lightning discharge and wave-particle interaction respectively are very well correlated.

As the wave is received at the low latitude Earth's station, Gulmarg, we may consider that the generation region could be near the equatorial plane of the field line corresponding to Gulmarg in the inner zone radiation belt $(\mathrm{L} \sim 1.2)$. In this region there are a large number of energetic electrons, which can effectively participate in the generation mechanism through Cyclotron resonance interaction (Rycroft 1972; Imhoff et al 1973). In order to explain the observed features of discrete VLF emissions we propose cyclotron resonance interaction between whistler mode wave and energetic electrons of inner radiation belt. We note that for effective transfer of energy from the interacting particle to the wave, resonance condition has to be satisfied which is written as

$$
1-\beta_{\|}=\frac{\omega_{H}}{\gamma \omega},
$$

where $\beta_{\|}=v_{\|} / c, v_{\|}$is the electron velocity along the magnetic field and $\gamma=\left(1-\beta_{\|}^{2}\right)^{-1 / 2}$. The refractive index for parallel propagating waves under condition $\omega^{2} \ll \omega_{H}^{-} \omega_{H}^{+}$is much greater than 1 and is written as (Stix 1962).

$$
n^{2}=\frac{\omega_{p e}^{2}}{\omega_{H e}\left(\omega+\omega_{H i}\right)},
$$


where $\omega_{p e} \omega_{H e}$ are the electron plasma frequency and electron gyrofrequency respectively. $\omega_{H i}$ is the proton gyrofrequency. The resonant energies $E_{\|}$for various frequencies of emissions can be written as (Tsurutani et al 1975)

$$
E_{\|}=\left(\frac{\omega_{H e}}{\omega_{p e}}\right)^{2}\left(\frac{\omega_{H e}}{\omega}\right)\left(1+\frac{\omega_{H i}}{\omega}\right) m_{0} c^{2},
$$

where $m_{0}$ is the rest mass of electron, $c$ is the velocity of light in vacuum.

The interacting waves can be amplified if the high-energy tail of the velocity distribution function has some finite anisotropy $A=\left(T_{\perp} / T_{\|}\right)-1$, where $T_{\perp}$ and $T_{\|}$are the temperatures of the electrons perpendicular to and parallel to the geomagnetic fields respectively. In the regime of linear theory, the growth rate of the wave for $\omega \ll \omega_{H e}$ is given by (Kennel and Pestschek 1966)

$$
\begin{aligned}
\Gamma= & \pi \omega_{H e}\left(1-\frac{\omega}{\omega_{H e}}\right)^{2} \frac{J\left(>E_{R}\right)}{2 V_{R} N_{T}} \\
& \times\left[A\left(V_{R}\right)-\frac{1}{\left(\omega_{H e} / \omega\right)-1}\right],
\end{aligned}
$$

where $J\left(>E_{R}\right)$ is the omnidirectional flux of electrons having energy greater than the resonance energy. $V_{R}$ is the resonance velocity and $N_{T}$ is the total number density of electrons.

\section{Results and discussions}

The observation of ELF/VLF emissions at lowlatitude station Gulmarg shows that, although discrete VLF emissions are rare under quiet conditions the occurrence rate of discrete VLF emissions is high during magnetic storms. We have recorded a new type of discrete emission during the magnetic storm period of 6-9 March, 1986. During substorms the inflow of the particles with energy of about $1 \mathrm{keV}$ from the plasma sheet into the inner magnetosphere takes place. Plasma is trapped by the geomagnetic field and accelerated. The oscillating particles drift to dawn (electrons) and to dusk (protons). The large-scale dawn-dusk electric field, which is increased during disturbed conditions cause plasma to drift across L shells. As electrons drift to dawn the conditions become more and more favourable for the cyclotron instability to arise (Bespalov and Trakhtengerts 1986) and the background plasma density increases from midnight to morning-noon hours owing to filling of the equatorial regions of the magnetosphere with cold ionospheric plasma. At a certain time the energetic electrons reach a certain region where the conditions for the cyclotron instability turn out to be

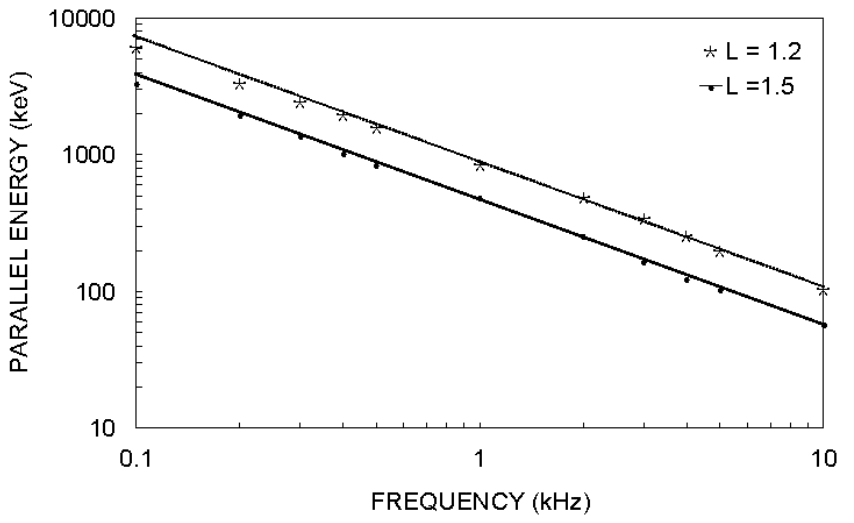

Figure 3. The variation of parallel energy of resonating electrons with wave frequency at $\mathrm{L}=1.2$ and 1.5.

optimal. In this region the dissipation of accumulated energy takes place by cyclotron instability. As a result of this dissipation the generation of ELF/VLF emissions and precipitation of resonant electrons into the loss-cone proceeds (Smirnova 1984).

In order to test cyclotron resonance interaction as a possible generation mechanism for discrete VLF emissions, we have computed the resonance energy of the high-energy interacting electrons and growth rate of whistler waves at $\mathrm{L}=1.2$ in the equatorial plane. The variations of parallel energy of resonating electrons with wave frequency for different L-values are shown in figure 3. It is found that the energy of resonating electrons decreases as L-value increases for a given frequency band. Burton and Holzer (1974) have shown that the discrete emissions are generated by cyclotron resonance with electrons in the energy range of 5$150 \mathrm{keV}$ with pitch angle distribution peaked at $90^{\circ}$ to $\mathbf{B}$ and anisotropy greater than a critical value. Further, it has been shown that the resonant energies for various frequencies of the emissions at $\mathrm{L}=1.2$ are in the $\mathrm{MeV}$ range (Lalmani et al 1972). Thus, the computed values of resonant energy for various frequencies are in good agreement with the reported results (Lalmani et al 1972; Burton and Holzer 1974).

Equation (4) shows that the wave growth linearly increases with anisotropy $A$ in the velocity distribution function. Further $\Gamma$ is positive only when $A>\omega /\left(\omega_{H e}-\omega\right)$. Thus there is a minimum anisotropy which is required for the wave to grow and hence for the generation of ELF/VLF emissions. The variations of this minimum anisotropy as a function of wave frequency for different Lvalues are shown in figure 4 . The anisotropy $A$ increases with frequency as well as L-value. It is also seen that the gyroresonance leading to extremely low frequency signals requires only small 


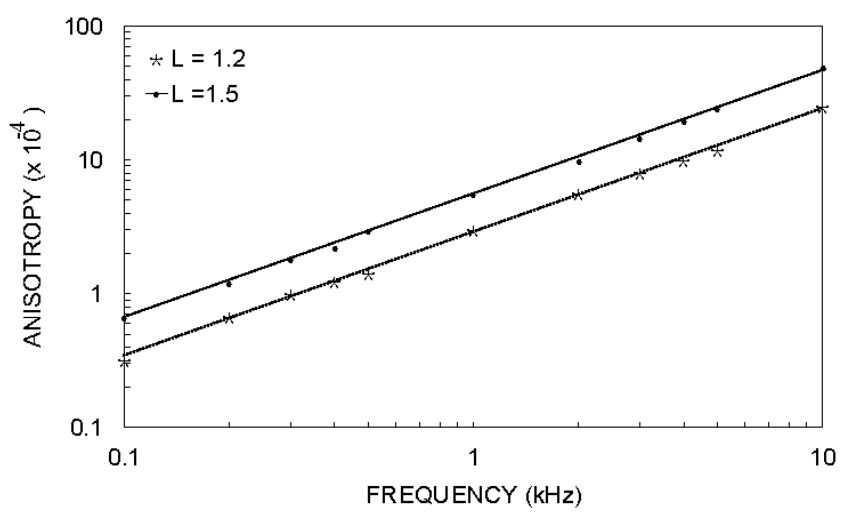

Figure 4. The variation of the minimum anisotropy as a function of wave frequency at $\mathrm{L}=1.2$ and 1.5.

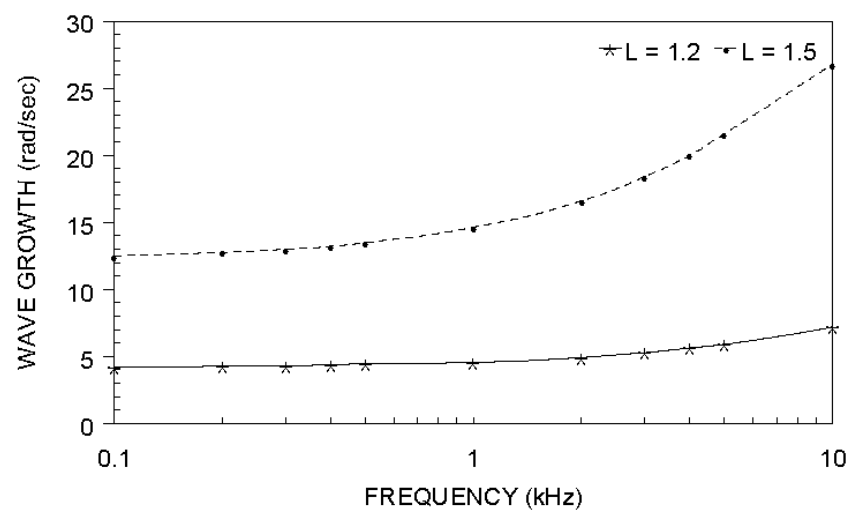

Figure 5. The variation of wave growth rate with excited frequency at $\mathrm{L}=1.2$ and 1.5 .

values of $A$, however on a particular field line, this requires resonance with electrons of much higher energy.

For computation of wave growth, the density of energetic electrons at different L-values are derived from the measurement of Katz (1966) who has reported the variation of electron flux as a function of energy for different L-values in the inner zone radiation belt. The variation of wave growth rate with excited frequency for $\mathrm{L}=1.2$ and $\mathrm{L}=1.5$ is shown in figure 5 . It is observed that the growth rate is larger and it increases with frequency. This large amplification causes significant enhancement in wave amplitude, which in turn triggers discrete emissions to be observed at low latitude ground station Gulmarg.

The rising frequency spectrum of discrete VLF emissions observed at Gulmarg can be explained by considering the interaction region to start from the equator and extend to some finite length in the southern hemisphere along the geomagnetic field line. After moving away from the equator, the local electron gyrofrequency becomes too large for the resonance condition to be still met and the generation of rising tone ceases. Thus, the maximum frequency of discrete emissions is controlled by the extension of interaction region along the geomagnetic field line.

In the above, we considered the generation of discrete emission through the process of wave-particle interaction in the magnetosphere. However, there is no direct evidence that these emissions belong to the magnetospheric origin. These emissions could be generated during lightning discharge along with tweeks. In this case it is not clear how to explain dispersion produced in the VLF signal called discrete emissions. The other possibility is that discrete emission is generated during instability in the ionosphere. Again this aspect has not been explored in detail.

\section{Conclusion}

A detailed spectral analysis of a new type of discrete VLF emissions observed at the low-latitude ground station Gulmarg during the strong magnetic activity period have been carried out. The possible generation mechanism for temporal and spectral features of these discrete VLF emissions is presented. It is suggested that these discrete emissions could be generated during resonant cyclotron interaction in the equatorial zone of the inner magnetosphere. A further experimental and theoretical study of discrete VLF emissions at low latitude would definitely contribute to a more detailed understanding of these phenomena.

\section{Acknowledgements}

This work is partially supported by the Department of Science and Technology (DST), New Delhi, Government of India and partly by UGC, New Delhi. (DS) thanks DST, under the BOYSCAST program (with reference SR/BY/A-19/05).

\section{References}

Bell T F, Inan U S and Helliwell R A 2000 Simultaneous triggered VLF emissions and energetic electron distributions observed on Polar with PWI and HYDRA; Geophys. Res. Lett. 27165.

Besplaov P A and Trakhtengerts V Y 1986 The cyclotron instability of the Earth's radiation belts; In: Review of Plasma Physics (ed.) Leontovich M A, Plenum, New York.

Burton R K and Holzer R E 1974 The origin and propagation of chorus in the outer magnetosphere; J. Geophys. Res. 791014.

Cornilleau-Wherlin N, Gendrin F, Lefeuvre F, Parrot M, Grand R, Jones D, Bahnsen A, Ungstrup E and Gibbons W 1978 VLF electromagnetic waves observed onboard GEOS-1; Space Sci. Rev. 22371. 
Dowden R L 1971 Electron energy spectrum and structure deduced from analysis of VLF discrete emissions by using the Helliwell criterion; J. Geophys. Res. 763034.

Hattori K, Hayakawa M, Lagoutte D, Parrot M and Lefeuvre F 1991 Further evidence of triggered chorus emissions from wavelets in the hiss band; Planet. Space Sci. 391465.

Hayakawa M 1989 Satellite observations of low latitude VLF radio noises and their association with thunderstorms; J. Geomag. Geoelectr. 41573.

Helliwell R A 1967 A theory of discrete emissions from the magnetosphere; J. Geophys. Res. 724773.

Imhoff W L, Gains E E and Regan J D 1973 Dynamic variations in intensity and energy spectra of electrons in the inner radiation belt; J. Geophys. Res. 784568.

Katz L 1966 Electron and proton observations; In: Radiation Trapped in Earth's Magnetic Field (ed.) Reidel D, Holland, p129.

Kennel C F and Pestschek H E 1966 Limit on stably trapped particle fluxes; J. Geophys. Res. 711.

Lalmani, Rao M, Somajajulu V V and Tantry B A P 1972 An analysis of multicomponent ground observation of VLF hiss, J. Geomag. Geoelecr. 24261.

Nunn D and Sazhin S S 1991 On the generation mechanism of hiss-triggered chorus; Ann. Geophys. 9603.

Rycroft M J 1972 VLF emissions in the magnetosphere; Radio Sci. 7811.

Santolik O and Gurnett D A 2003 Transverse dimensions of chorus in the source region; Geophys. Res. Lett. 30 1031, doi: 1029/2002GL016178.

Santolik O, Gurnett D A, Pickett J S, Parrot M and Cornilleau-Wehrlin N 2004 A microscopic and nanoscoipic view of storm time chorus on 31 March, Geophys. Res. Lett. 31 L02801, doi:10.1029/2003 GLO18757.

Sazhin S S and Hayakawa M 1992 Magnetospheric chorus emissions: A Review; Planet. Space Sci. 40(5) 681.

Shvets A V and Hayakawa M 1998 Polarization effects for tweek propagation; J. Atmos. Solar Terr. Phys. 60 461.

Singh A K and Singh R P 1996 Propagational features of higher harmonic tweaks at low latitudes; Earth Moon and Planets $\mathbf{7 3} 277$.

Singh A K and Singh R P 2004 Observation of discrete VLF emissions at low latitudes and their generation mechanism; Earth Planets Space 56 1067-1074.

Singh R, Patel R P, Singh R P and Lalmani 2000 An experimental study of hiss-triggered chorus emissions at low latitude; Earth Planets Space 5237.

Siingh, Devendraa, Shubha Singh and Singh R P 2005 Study of wave particle interaction in disturbed magnetosphere; Indian J. Radio Space Physics 34 305-311.

Smirnova N A 1984 Fine structure of the ground observed VLF chorus as an indicator of the wave-particle interaction process in the magnetosphere; Planet. Space Sci. 32 425 .

Stix T H 1962 The theory of plasma waves; Mc Graw-Hill, New York.

Trakhtengerts V Y 1999 A generation mechanism for chorus emission; Ann. Geophys. 1795.

Tsurutani B T, Smith E J and Thorne R M 1975 Electromagnetic hiss and relativistic electron losses in the inner zone; J. Geophys. Res. 80600. 